\title{
Dispersion EPR: Considerations for Low-Frequency Experiments
}

\author{
James S. Hyde ${ }^{1} \cdot$ Robert A. Strangeway $^{1,2} \cdot$ Jason W. Sidabras $^{1}$ (D)
}

Received: 16 March 2021 / Revised: 28 April 2021 / Accepted: 12 May 2021 /

Published online: 29 May 2021

(c) The Author(s) 2021

\begin{abstract}
The hypothesis is made that the dispersion electron paramagnetic resonance (EPR) spectrum can yield a higher signal-to-noise ratio than the absorption spectrum in diagnostic examinations if phase noise in the bridge is under control. The rationale for this hypothesis is based on the observation that the dispersion spectrum becomes more intense than the absorption spectrum at high incident powers. The rationale is dependent on optimization of high microwave efficiency $\left(\Lambda ; \mathrm{mT} / \mathrm{W}^{1 / 2}\right)$ and low-quality factor ( $Q$-value) sample resonators as well as the use of microwave sources with reduced phase noise. Microwave frequencies from 1.2 to $94 \mathrm{GHz}$ are considered. Although the dispersion display appears to be observable with an adequate signalto-noise ratio for most EPR research initiatives, a weakness of microwave bridges for studies at high incident microwave power was identified. Spurious leakage of incident microwave power through the circulator, thereby bypassing the probe leading to the resonator, can result in a decreased signal-to-noise ratio in both absorption and dispersion because of phase noise. For dispersion EPR with low $Q$-value sample resonators, this leakage is the primary contributor to phase noise at the receiver. In this work, we focus on the design of microwave reflection bridges and discuss possible methods to ameliorate this source of noise.
\end{abstract}

\section{Introduction}

In continuous wave EPR spectroscopy, the dispersion component of the signal is seldom observed because of high levels of phase noise arising from the microwave source. When the incident microwave power is increased to saturating conditions, the absorption signal broadens and loses intensity while the dispersion may not be

Jason W. Sidabras

jwsidabras@mcw.edu

1 Department of Biophysics, Medical College of Wisconsin, Milwaukee, WI, USA

2 Department of Electrical Engineering and Computer Science, Milwaukee School of Engineering, Milwaukee, WI, USA 
affected until much higher powers. Under these conditions, detecting dispersion is advantageous relative to absorption. An overall increase in dispersion signal intensity relative to the maximum absorption signal intensity will occur [1]. Situations can be encountered where detection of dispersion is desirable and even necessary because absorption is not readily observable. Two examples are briefly reviewed here.

The first comprehensive electron-nuclear double resonance (ENDOR) experiment was reported by Feher in 1959 [2]. He investigated the structures of various impurities in silicon, and later described ENDOR experiments in biological samples of myoglobin [3] and chlorophyll [4]. The silicon experiments were performed at $\mathrm{X}$-band (nominally $9.5 \mathrm{GHz}$ ) at a temperature of $1.25 \mathrm{~K}$, while the biological samples were performed at $2 \mathrm{~K}$ and $70 \mathrm{~K}$, respectively. Spin lattice relaxation times were very long, and electron paramagnetic resonance (EPR) absorption signals may not have been observable since none were shown. All experiments were carried out by observing the dispersion EPR signal under conditions of adiabatic rapid passage. A magnetic field modulation frequency of $100 \mathrm{~Hz}$ was used for detection. Nuclear magnetic resonance was detected indirectly by observing the change in the EPR dispersion signal as a function of the irradiating radiofrequency.

Another example of the use of dispersion is the experiment of Hyde and Dalton, which is today called "saturation transfer spectroscopy," or STEPR [5]. A nitroxide radical spin label was attached to a macromolecule undergoing very slow rotational diffusion. In the range of rotational correlation times of $10^{-3}-10^{-6} \mathrm{~s}$, absorption EPR spectra appeared to be in the rigid limit. The spectra in the dispersion mode, however, changed dramatically. These experiments were carried out with $100 \mathrm{kHz}$ field modulation. The rotational correlation time effects arose under conditions of adiabatic rapid passage, as in the Feher experiment, even though the sample was fluid and at physiological temperatures. Observation of macromolecular motion in this slow rotational diffusion range using spin labels had not previously been possible with conventional techniques.

It soon became apparent that excess phase noise in the dispersion mode was limiting the application of STEPR to biological systems. It was subsequently discovered that passage effects could be observed in an unusual display - the second harmonic absorption with the lock-in detector set out-of-phase. Signals were smaller, but the signal-to-noise ratio was larger [6]. This method has become the standard of practice. An early review by Thomas and Hyde provides background on the details of the methodological development [7].

The experiment of Hyde and Dalton provides additional motivation to implement phase noise reduction techniques in EPR bridges, especially for dispersion mode experiments. At sufficiently low non-saturation power levels, the absorption and dispersion modes can be converted through a Hilbert transformation. Detection of both yields $2^{1 / 2}$ increase in the signal-to-noise ratio, which can be helpful when using oxygen-sensitive probes with narrow lines. However, at high powers, these displays contain differing information.

The use of low-frequency modulation $(27 \mathrm{kHz})$ was a strategic decision by Swartz et al. for clinical [8] and field-deployable L-band bridges [9] to reduce artificial broadening from passage effects. Oximetry probes, such as lithium phthalocyanine, 
have linewidths as low as $14 \mathrm{mG}$ in the absence of oxygen [10]. To realize such narrow linewidths the field modulation frequency must be reduced to meet the requirement of

$$
f_{\text {mod }} / \Delta H_{l w}<2.8 \mathrm{MHz} / \mathrm{G},
$$

where $f_{\text {mod }}$ is the field modulation frequency and $\Delta H_{l w}$ is the linewidth of the sample [11]. However, at lower field modulation frequencies, the phase noise of the source is elevated, which can reduce the signal-to-noise ratio of the dispersion signal. This problem is exacerbated when high quality factor ( $Q$-value) sample resonators are utilized.

For this reason, the absorption mode is generally detected. However, Feher's early experiment indicates that if it became possible to observe dispersion without excess noise, an increase in the signal-to-noise ratio could be achieved relative to the observation of the absorption mode. When the system is easily saturable, the increase could be significant. This is a strong rationale for developmental efforts to reduce phase noise and detect in the dispersion mode.

In this paper, we first consider the complex-valued reflection coefficient and the phase noise incident on a sample resonator. This theoretical development is then used to interpret experimental noise measurements using a Q-band (nominally $35 \mathrm{GHz}$ ) EPR bridge in the dispersion mode. The choice of resonator greatly affects the results. The rational for sample resonators with high resonator efficiency parameter $\Lambda\left(\mathrm{mT} / \mathrm{W}^{1 / 2}\right)$ and low $Q$-value is addressed, where $\Lambda$ is the value of the RF field that is produced at a point in the sample when $1 \mathrm{~W}$ of microwave power is incident on a matched resonator. Previous phase noise results from W-band (nominally $94 \mathrm{GHz}$ ) experiments are examined in the context of a hypothesis that microwave leakage through the directional coupler that interfaces the sample resonator probe to the EPR bridge can affect results in the dispersion mode. X-band (nominally $9.5 \mathrm{GHz}$ ) test measurements are presented regarding the leakage hypothesis. Finally, recommendations are made to reduce phase noise at the receiver in low-frequency bridges $(1.2 \mathrm{GHz})$.

\section{Theory}

Early work in the literature assumed that the bridge consists of a single fundamental oscillator input into a bridge circuit and that the sample is placed in a resonator with a high $Q$-value. As bridge systems become more complicated and the loop-gap resonator (LGR) or similar resonators came into use, the phase noise assumptions were no longer valid. These assumptions were: phase noise demodulation by the sample resonator dominated the received noise in dispersion, and a $100 \mathrm{kHz}$ field modulation frequency was required for sufficiently low source phase noise at that offset from the carrier frequency. A well-known reflection coefficient analysis is revisited with the assumption of low $Q$-value microwave resonators. A resonator coupled to a transmission line results in the following reflection coefficient $\Gamma$ 


$$
\Gamma=\frac{(\beta-1)-i Q_{o} \frac{2 \Delta \omega}{\omega_{o}}}{(\beta+1)+i Q_{o} \frac{2 \Delta \omega}{\omega_{o}}},
$$

where $\omega_{o}$ is the resonant frequency of the resonator, $\Delta \omega$ is a frequency offset from $\omega_{o}, Q_{o}$ is the $Q$-value of the resonant circuit without transmission line loading, and $\beta$ is the coupling factor [12-14]. When the resonator is critically coupled, $\beta$ is unity. At resonance, the reflection coefficient $\Gamma_{o}$ and return loss $\mathrm{RL}_{o}$ are related to the coupling factor by

$$
\Gamma_{o}=\Gamma\left(\omega_{o}\right)=\frac{\beta-1}{\beta+1} \quad \text { and } \quad \operatorname{RL}_{o}=\operatorname{RL}\left(\omega_{o}\right)=-20 \log \left|\Gamma_{o}\right|=20 \log \left|\frac{\beta+1}{\beta-1}\right| .
$$

Finally, the reflection coefficient expression can be rearranged into real and imaginary parts, such that,

$$
\Gamma=\Gamma_{\operatorname{Re}}+i \Gamma_{\operatorname{Im}}=\frac{\left(\beta^{2}-1\right)-\left(Q_{o} \frac{2 \Delta \omega}{\omega_{o}}\right)^{2}}{(\beta+1)^{2}+\left(Q_{o} \frac{2 \Delta \omega}{\omega_{o}}\right)^{2}}-i \frac{2 \beta\left(Q_{o} \frac{2 \Delta \omega}{\omega_{o}}\right)}{(\beta+1)^{2}+\left(Q_{o} \frac{2 \Delta \omega}{\omega_{o}}\right)^{2}} .
$$

Now, consider a low $Q$-value resonant circuit at relatively low-frequency offsets; then $(\beta+1)^{2}>>\left(Q_{o} \frac{2 \Delta \omega}{\omega_{o}}\right)^{2}$ and the reflection coefficient simplifies to

$$
\Gamma=\frac{\left(\beta^{2}-1\right)-\left(Q_{o} \frac{2 \Delta \omega}{\omega_{o}}\right)^{2}}{(\beta+1)^{2}}-i \frac{2 \beta\left(Q_{o} \frac{2 \Delta \omega}{\omega_{o}}\right)}{(\beta+1)^{2}} .
$$

When the EPR bridge is set to dispersion and the resonator is matched at resonance $(\beta \approx 1)$, the imaginary part of the reflection coefficient becomes

$$
\Gamma_{\mathrm{Im}} \approx-i\left(Q_{o} \frac{\Delta \omega}{\omega_{o}}\right) .
$$

The principles behind the demodulation of phase noise by reflection from a resonator were described by Ashley et al. [15] and Ondria [16] in the context of phase noise measurements. The power reflected from the resonator is directly proportional to the square of the offset frequency from the carrier, $\Delta \omega^{2}$. Conversely, the phase noise power density to carrier ratio $(\mathrm{dBc} / \mathrm{Hz})$ from a fundamental microwave source is inversely proportional to $\Delta \omega^{2}$ over a certain offset frequency range [17]. This offset frequency range generally spans from where $1 / f$ noise effects are present (on the order of $\mathrm{kHz}$, but highly device dependent) to where white noise sets the thermal noise floor $(-174 \mathrm{dBm} / \mathrm{Hz}$ plus the active device noise figure). Hence, to the first order within this offset range, the suppression of phase noise sidebands by a low Q-value microwave resonator is counterbalanced by the increase in phase noise as offset frequency decreases. If this was the only source of phase noise demodulation in the bridge, the selection of the field modulation frequency could be lower than the 
traditional $100 \mathrm{kHz}$ but somewhat above $1 / f$ frequencies of the active components in the bridge and characteristic acoustic frequencies of the environment.

\section{Results and Discussion}

\subsection{Resonator Considerations}

At lower microwave frequencies, the rationale of the LGR is to improve signal intensity for samples of limited volume. However, an interesting finding from Sidabras et al. is that at W-band, a $\mathrm{TE}_{011}$ cylindrical cavity has the same concentration sensitivity as a five-loop-four-gap LGR [18] when the microwave fields incident on the sample are adjusted to the same value. For the same sample volume and incident power, the LGR had an order of magnitude higher concentration of magnetic stored energy in the sample compared to the $\mathrm{TE}_{011}$ cavity. Conversely, the LGR $Q$-value was an order of magnitude lower than the $\mathrm{TE}_{011}$ cavity [19]. This presents an opportunity to re-think resonator design in the context of dispersion EPR.

One possible alternative approach to improve EPR detection of dispersion is to use a bimodal LGR. In the absence of magnetic resonance, the two modes are ideally isolated. The incident microwave power is in one mode and isolated from the other. When magnetic resonance occurs, a signal is induced in the second mode which is detected at the receiver. Tsapin et al. [20] and Rinard et al. [21-24] have described bimodal sample resonators. However, in this paper we only consider single-mode sample resonators configured for use in conventional reflection bridges. The benefits of a LGR for use in the detection of the dispersion mode were first reported by Hyde, Froncisz, and Kusumi [25].

When focusing on dispersion detection, the resonator must have a high-efficiency parameter $\Lambda$ and a low $Q$-value. Both characteristics contribute to decreased demodulation of phase noise. For example, the increase in $\Lambda$ results in the same magnetic stored energy in the sample at lower microwave power, decreasing received noise. A low $Q$-value has the benefit of lower phase noise demodulation reflecting from the sample resonator to the receiver.

\subsection{Q-Band}

Microwave phase noise measurements using the Varian Q-band (nominally $35 \mathrm{GHz}$ ) EPR microwave bridge have been published [26], and these measurements were later extended [27]. An LGR at $35 \mathrm{GHz}$ was designed and phase-noise measurements made comparing the LGR with the commercial Varian $\mathrm{TE}_{011}$ cavity [26].

In general, microwave phase noise dominates at high microwave power incident on the resonator, and receiver noise dominates at low microwave power. Data shown with a klystron as the microwave source and a point contact 1 N53D diode as the detector in the microwave bridge were obtained and plotted [26]. Shown in Fig. 1, the dispersion mode was used to highlight the phase noise and receiver noise for two sample resonators. The ordinate is microwave voltage in arbitrary units. Breakpoints 
Fig. 1 Noise of the Q-band bridge in dispersion mode as a function of main power attenuation. Dashed line indicates theoretical improvement. Solid lines reproduced from Ref. [26], with the permission of AIP Publishing

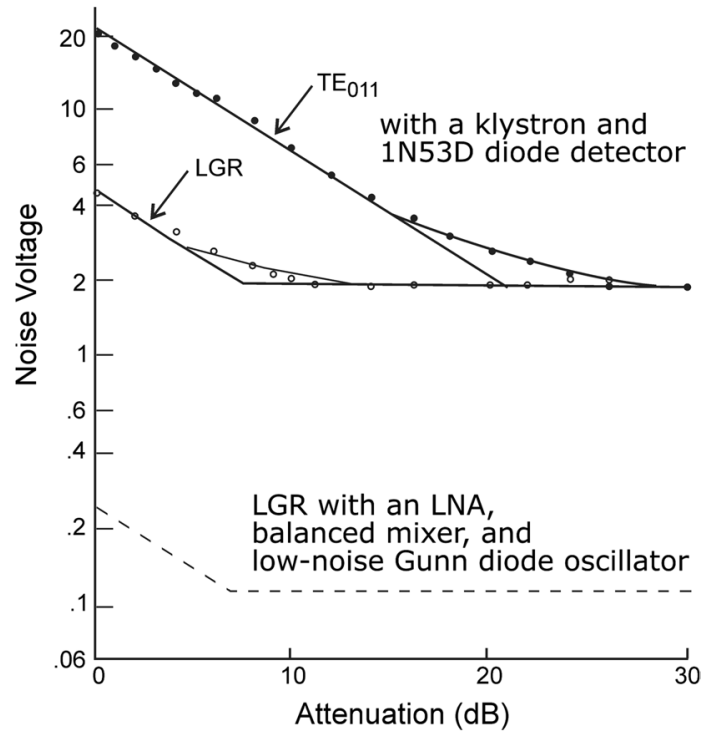

are observed where the limiting noise shifts between the microwave source and the microwave detector. One expects the difference of break-point powers $(13 \mathrm{~dB})$ to be given by

$$
20 \log \left[\frac{Q_{o}\left(\mathrm{TE}_{011}\right)}{Q_{o}(\mathrm{LGR})}\right],
$$

which was calculated to be $17 \mathrm{~dB}$ for Q-band in Ref. [26] (agreement was adequate based on microwave measurement technology in 1986). We can conclude that at equal incident power levels, the LGR exhibits a significant advantage in noise profile with respect to the $\mathrm{TE}_{011}$ cavity.

The ratio of the resonator efficiency of the LGR that was used in Ref. [26] to the $\mathrm{TE}_{011}$ cavity is 3.1 , or about $10 \mathrm{~dB}$. Thus, if the comparison of Fig. 1 were shifted to hold the peak microwave magnetic field in the two resonators at the same value for the same incident power, the LGR breakpoint must be moved nearly to the origin. The combined benefits of low $Q$-value and high $\Lambda$ of the LGR nearly solved the problem of excess phase noise when the microwave bridge used a klystron for a microwave source and a point-contact diode for detection.

Further modifications made in the bridge included a low noise microwave amplifier (LNA), a balanced mixer, and a custom-designed low noise Gunn diode oscillator coupled to a high $Q$-value microwave cavity [27-29]. The bridge is configured to permit convenient switching between absorption and dispersion. The addition of an LNA and balanced mixer were found to improve the receiver noise figure by $24.7 \mathrm{~dB}$. Implementing the low phase noise Gunn diode oscillator reduced the microwave phase noise at $100 \mathrm{kHz}$ from the carrier by $26 \mathrm{~dB}$ [27]. A theoretical curve for the LGR has been drawn (dashed) on Fig. 1 for these modifications. The benefits of the LGR in combination with an LNA, a balanced mixer, and a low phase 
A

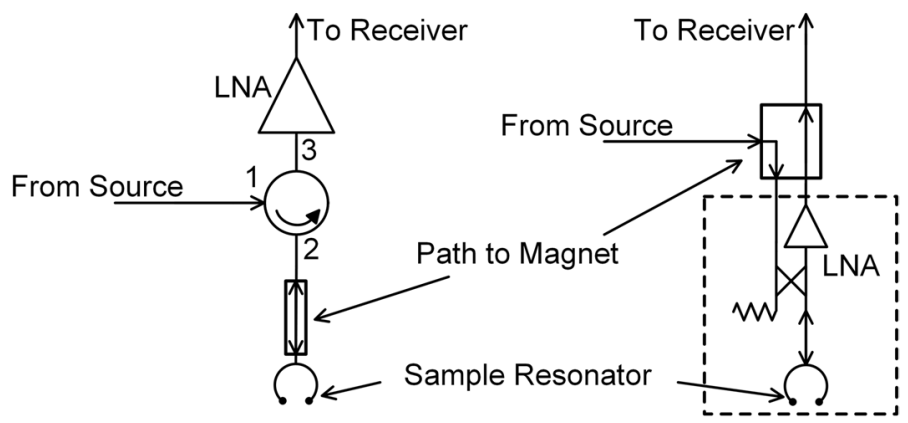

Fig. 2 A Bridge setup with a circulator and a path to the magnet. Microwave leakage occurs from Port 1 to 3. B Proposed bridge setup for preclinical and clinical EPR with the directional coupler (e.g., $10 \mathrm{~dB}$ coupling factor) moved adjacent to the sample resonator and the EPR signal directly fed to an LNA

noise microwave fundamental source resulted in a very significant advance when carrying out EPR studies in the dispersion mode at Q-band.

\subsection{W-Band}

Additional phase noise observations were made in a custom-designed W-band spectrometer [30-32]. In this instrument, low phase noise 35 and $59 \mathrm{GHz}$ sources are mixed to generate low phase noise $94 \mathrm{GHz}$ incident power. A five-loop-four-gap LGR was employed with an efficiency of $1.1 \mathrm{mT} / \mathrm{W}^{1 / 2}$ and a $Q_{o}$ of approximately 180 with an aqueous sample [18]. At W-band, the small size of the LGR lends itself naturally to the combination of low $Q$-value and high $\Lambda$ required for dispersion operation.

In a continuous wave EPR experiment with this spectrometer, $433 \mathrm{~Hz}$ field modulation resulted in significantly lower noise in dispersion than expected [31]. The bridge arms were balanced, and thus delay line demodulation of phase noise from the resonator reflection path was negligible. The unloaded $Q$-value of the LGR with an aqueous sample was 180 . From Eq. 6, using $Q_{o}=180,94 \mathrm{GHz}$, and $433 \mathrm{~Hz}$ offset, the return loss was calculated to be $121 \mathrm{~dB}$. Thus, phase noise sidebands from the source would also be suppressed at $121 \mathrm{~dB}$ at $433 \mathrm{~Hz}$ offset from a $94 \mathrm{GHz}$ carrier assuming this was the only means for phase noise to be present at the receiver in dispersion.

However, measurements indicated that a significant leakage level was present in the W-band bridge despite the use of a directional coupler of moderate directivity [31]. Consequently, there is also a significant leakage of phase noise to the receiver. From a typical bridge setup, illustrated in Fig. 2A, the source power enters the circulator (Port 1), travels to the sample resonator (via Port 2), back to the circulator, and to the LNA (Port 3). Here, leakage is defined as the power that couples directly from Port 1 to Port 3 and into the LNA input. Phase noise that leaks directly to the signal receiver is not diminished by the reflection coefficient of the low $Q$-value sample resonator. As such, phase noise is present at the receiver in both $I / Q$ channels, in 
Table 1 Resonator return loss vs. leakage calculations

\begin{tabular}{llll}
\hline $\begin{array}{l}\text { Frequency offset } \\
\text { from } f_{o}(\mathrm{kHz})\end{array}$ & $\begin{array}{l}\mathrm{RL}(\mathrm{dB}) \text { due } \\
\text { to } \Gamma_{\mathrm{Re}}\end{array}$ & $\begin{array}{l}\mathrm{RL}(\mathrm{dB}) \text { due } \\
\text { to } \Gamma_{\mathrm{Im}}\end{array}$ & $\begin{array}{l}\text { Leakage }(-\mathrm{dB}) \\
1\end{array}$ \\
\hline 10 & 82 & 30 \\
10 & 30 & 62 & 30 \\
100 & 30 & 42 & 30 \\
\hline
\end{tabular}

general, even at low microwave power levels. With a high $Q$-value sample resonator, the phase noise of the bridge is maximally demodulated by the sample resonator, but with a low $Q$-value sample resonator, the leakage can dominate.

For these experiments, the phase shifter in the reference arm, which is in the receiver, is set to detect dispersion of the EPR signal from the sample resonator. However, the phase of the leakage path length is not set for dispersion, in general, because the leakage path is not balanced with the signal path. Therefore, with a low $Q$-value resonator, the phase noise of the source can be present in both signal channels (absorption and dispersion).

In summary, the leakage of a signal through a circulator (Fig. 2A) or directional coupler (Fig. 2B) that interfaces the bridge to the sample probe is often on the order of the reflection from the real part of the reflection coefficient of the sample resonator. Furthermore, the leakage can be significantly larger than the reflection from the imaginary part of the reflection coefficient of a low $Q$-value sample resonator. This leakage directly limits the detection sensitivity of the bridge and produces significant offsets when frequency modulation is employed [30]. We note that positioning the coupler adjacent to the sample resonator reduces the difference in the leakage path and signal path which further diminishes delay-line demodulation for the unbalanced path. This phenomenon can be expected to occur to varying degrees at other microwave frequencies.

\subsection{X-Band}

Early in the history of LGR development, it was recognized that this structure resulted in improved measurement of dispersion spectra in routine studies at X-band [25]. A robust capability for acquisition of dispersion spectra at this frequency is obviously highly desirable. Analysis and experimental studies of leakage at X-band have been carried out.

Sample calculations for a two-loop-one-gap LGR by Molecular Specialties are given in Table 1. The resonant frequency here is $9.6 \mathrm{GHz}$, and the $Q_{o}$ with no sample is 728. Assume a return loss at the sample resonator center frequency of $30 \mathrm{~dB}$ and a leakage through the circulator that interfaces to the resonator of $30 \mathrm{~dB}$, which is typical of a waveguide circulator in this frequency range and is generally considered good for a coaxial circulator (the isolation specification for most coaxial circulators is between 20 and $30 \mathrm{~dB}$ ). It is evident that with this somewhat low $Q$-value resonator, the real part of the reflection coefficient dominates and is on the order of the leakage. The imaginary part is mostly negligible relative to the leakage. If the 


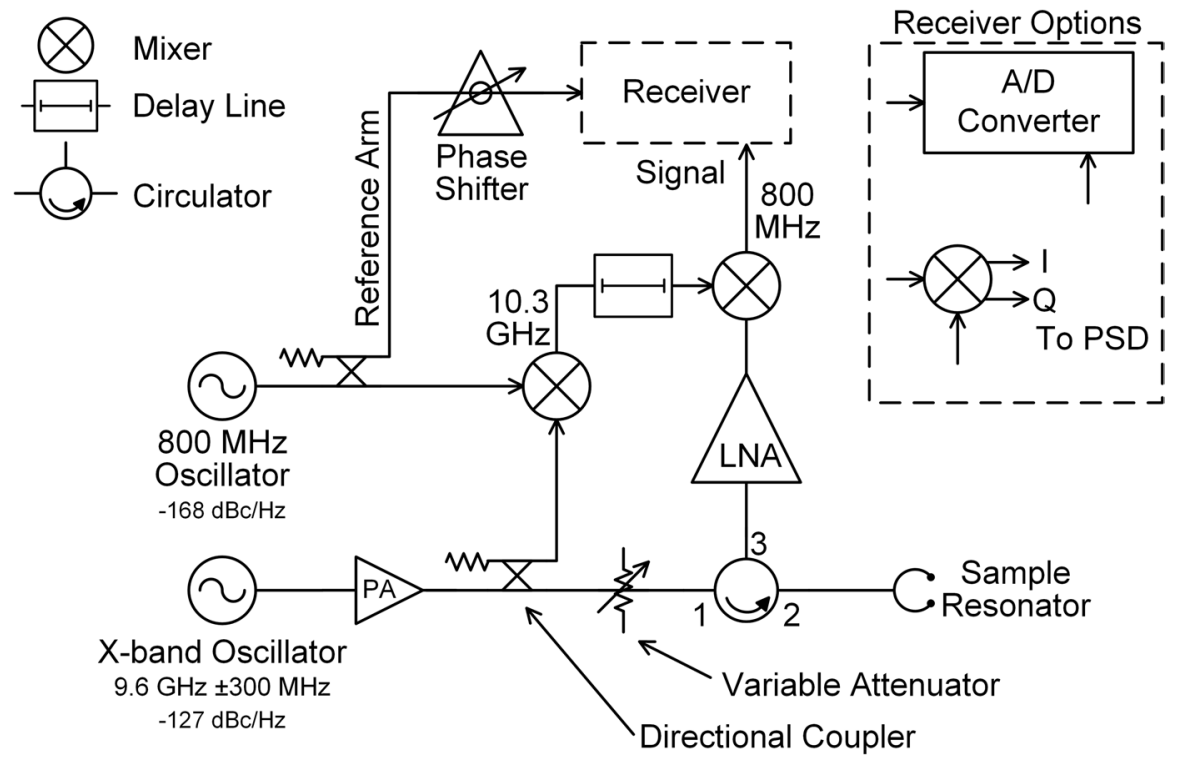

Fig. 3 An X-band bridge was designed with an $800 \mathrm{MHz}$ receiver for direct detection. The bridge is path-length equalized and balanced in the delay line arm (nominally $10.3 \mathrm{GHz}$ ). Two receiver options are available: direct detection of $800 \mathrm{MHz}$ or $I / Q$ mixer to phase-sensitive detector (PSD). Both microwave sources are independent fundamental oscillators

return loss of the resonator at microwave resonance and/or the leakage is higher, the leakage level becomes larger than both parts of the reflection coefficient.

The $Q$-value of this LGR decreases significantly once loaded with a sample, further increasing the return loss due to the imaginary part of the reflection coefficient. Thus, the imaginary part of the reflection coefficient does not contribute significantly to received phase noise relative to the phase noise in the leakage signal and reflected signal due to the real part of the reflection coefficient. A bridge test (not an EPR experiment) was performed to verify this assertion outlined in Table 1. The bridge in Fig. 3 is a custom-designed X-band bridge used in non-adiabatic rapid sweep spectroscopy [33]. The X-band microwave source is an yttrium iron garnet (YIG) tuned oscillator (Teledyne; FS3044R) with a measured maximum phase noise of $-127 \mathrm{dBc} / \mathrm{Hz}$ at $100 \mathrm{kHz}$ offset. The source was frequency modulated (FM) to emulate phase noise sidebands at $1 \mathrm{kHz}$, then $10 \mathrm{kHz}$, and finally $100 \mathrm{kHz}$ offset frequencies from the carrier. The FM sidebands in each case were $50 \mathrm{~dB}$ down from the carrier. Initially, a termination was placed on Port 2 of the circulator. Introduction of the $800 \mathrm{MHz}$ circuitry, see Fig. 3, permits use of a phase-sensitive detector or other digital detection equipment. The phase in the $800 \mathrm{MHz}$ reference arm was adjusted for a maximum signal at the quadrature output of the $I / Q$ mixer. The output of the mixer was virtually the same voltage at all three FM frequencies, as expected.

Then, the LGR was attached to Port 2 of the circulator though a $1 \mathrm{~m}$ coaxial line. The resonator match was adjusted to minimize the microwave power as detected after the LNA. It should be noted that the minimized power is the complex sum of 
the reflected signal and the leakage signal, not solely the former. The phase in the reference arm was readjusted for maximum voltage at the quadrature output of an $I / Q$ mixer. Again, the output of the mixer was virtually the same at all three FM frequencies, although the absolute level differed from the test with the termination. This aspect is due to the unknown complex summation of the leakage and the residual reflection from the termination, which if inversely phased, could result in a significantly lower absolute power level. The primary observations are that the signal at the mixer output did not change over the $1-100 \mathrm{kHz}$ offset frequency and that the imaginary part of the reflection coefficient of the low $Q$-value resonator did not result in observable elevated FM demodulation as the offset frequency increased. This would not be the case with a high $Q$-value sample resonator. This test measurement provides evidence for the presence of leakage.

Additional reasons for the presence of phase noise in both $I / Q$ channels are present. The difference in the signal path for the reflected signal from the resonator and the signal path of the leakage results in a total signal phase that differs from either one. Resonator matching by minimizing the complex sum of these two signals can result in an offset of the microwave carrier frequency from the resonant frequency of the sample resonator. Consequently, each channel of the $I / Q$ mixer can have phase noise components phased for demodulation. Every coaxial connection also has a return loss, on the order of $30 \mathrm{~dB}$ for high-quality coaxial connectors, but often worse, which results in unknown phase relationships between the various sources of reflected and leakage signals. It is surmised that sample resonator frequency discrimination is not the primary cause of phase noise demodulation in a bridge with a low $Q$-value sample resonator. It is concluded from these experiments that reducing the phase noise of the microwave source will have the largest impact on reducing overall phase noise effects at X-band. This microwave frequency is appropriate to test the hypothesis that there exists a range of conditions at high incident microwave power where the dispersion display is significantly more intense than the absorption display.

\subsection{L-Band}

Dispersion experiments at low microwave frequencies are considered here. Leakage can be reduced by replacing the circulator with a high-directivity directional coupler positioned adjacent to the sample resonator, illustrated in Fig. 2B [31]. At lower microwave frequencies, circulators may be especially problematic due to lower isolation relative to waveguide circulators. At low frequencies, direct detection of the carrier is available. Ahmad et al. working in the group of Zweier designed a direct detection system at L-band for EPR oximetry and imaging [34]. The system collects multiple harmonics of the field modulation, both in-phase and out-of-phase with respect to the field modulation. A novel method of processing signals in the presence of phase noise is presented. Kozioł et al. working in the group of Froncisz describe a novel instrument that was designed using direct digital synthesis technology for the explicit purpose of improved acquisition of diagnostic EPR information [35]. The work was evaluated using a surface coil and samples that were inspired by 
Table 2 Typical phase noise for a few oscillators commercially available and in EPR literature

\begin{tabular}{|c|c|c|c|c|}
\hline Frequency & Tuning range & Phase noise & Technology & References \\
\hline $9.6 \mathrm{GHz}$ & $\pm 300 \mathrm{MHz}^{\dagger}$ & $\begin{array}{l}-127 \mathrm{dBc} / \mathrm{Hz} @ 100 \mathrm{kHz} \\
-102 \mathrm{dBc} / \mathrm{Hz} @ 10 \mathrm{kHz}\end{array}$ & YIG Oscillator $(8.8-9.9 \mathrm{GHz})$ & $\ddagger$ \\
\hline $1.15 \mathrm{GHz}$ & $\pm 25 \mathrm{MHz}$ & -142 dBc/Hz@100kHz & $\begin{array}{l}\text { Direct Digital Synthesis with } \\
10 \mathrm{MHz} \text { reference }\end{array}$ & [35] \\
\hline $1.2 \mathrm{GHz}$ & $\pm 20 \mathrm{MHz}$ & -135 dBc/Hz@100kHz & $\begin{array}{l}\text { Coaxial Resonator Based Oscil- } \\
\text { lators }\end{array}$ & $\S$ \\
\hline $1.3 \mathrm{GHz}$ & $\pm 135 \mathrm{MHz}$ & -123 dBc/Hz@100kHz & Voltage Controlled Oscillator & $* *$ \\
\hline $800 \mathrm{MHz}$ & $\begin{array}{l}\text { (fixed oscil- } \\
\text { lator) }\end{array}$ & -168 dBc/Hz@100kHz & SAW Clock Oscillator & $\dagger \dagger$ \\
\hline \multicolumn{5}{|c|}{ Division by $N$} \\
\hline $1.2 \mathrm{GHz}$ & $\pm 75 \mathrm{MHz}$ & -145 dBc/Hz@100 kHz & $\begin{array}{l}\text { 9.0-10.2 GHz YIG Oscillator } \\
\text { divided by } 8\end{array}$ & \\
\hline
\end{tabular}

\footnotetext{
As currently limited in the bridge.

Teledyne model FS3044R.

${ }^{\S}$ Crystek Corp. model CVCO55CCQ

${ }^{* *}$ Crystek Corp. model CVCO55CL-1220-1490, which has the same characteristics as the oscillator in Ref. [34]

${ }^{\dagger \dagger}$ Crystek Corp. model CCSO-914X SAW Clock Oscillator
}

the on-going research of Harold M. Swartz. Dispersion and absorption spectra of comparable signal-to-noise ratios were obtained at $1.15 \mathrm{GHz}$ and $30 \mathrm{~mW}$ of incident power. Spectra were combined resulting in an improved signal-to-noise ratio. One general recommendation of these two papers (and others, such as Tseitlin et al. [36]) is to acquire all information available-dispersion and absorption, both in-phase and out-of-phase with respect to field modulation.

A fundamental oscillator generally trades off phase noise performance for tunability. Frequency division is a well-known technique to reduce phase noise from a source by $20 \log _{10}(N)$, where $N$ is the frequency division factor. Under this principle, the tuning range of the oscillator is also reduced by $N$. However, higher frequency fundamental oscillators generally have wider tuning ranges that remain adequate when divided by $N$. Frequency division of fundamental sources presents an opportunity to lower phase noise with sufficient overall frequency tunability.

The phase noise of a few select fundamental oscillators that are either customdesigned or commercially available are shown in Table 2 . Phase noise reduction by frequency division is also illustrated. For low-frequency EPR, an X-band YIG oscillator followed by frequency division (factor of 8) gives superior phase noise performance with a $\pm 75 \mathrm{MHz}$ tuning range compared to typical fundamental sources, see Table 2. The residual phase noise of frequency dividers is about $-150 \mathrm{dBc}$ at $100 \mathrm{kHz},{ }^{1}$ so a larger frequency division ratio would result in marginal system phase noise improvement and require a larger power amplifier gain.

\footnotetext{
$\overline{1}$ Analog Devices model ADF5002.
} 


\section{Summary}

This paper began with an observation from the literature that in selected circumstances the continuous wave EPR dispersion signal can be significantly higher than the absorption signal. The hypothesis was made that there could be a major opportunity for improvement of signal-to-noise ratio in diagnostic applications by observation of the dispersion signal at high microwave power.

Reduction of phase noise of the source at L-band is felt to be a promising direction for future development of technology for diagnostic EPR. For instance, by utilizing an X-band YIG source and frequency division to produce a nominal $1.2 \mathrm{GHz}$, a system with a theoretical phase noise of $-145 \mathrm{dBc} / \mathrm{Hz}$ at $100 \mathrm{kHz}$ with a tuning range of $\pm 75 \mathrm{MHz}$ can be realized. In addition to obvious efforts to lower the phase noise of the microwave source, we have identified principles that can further reduce system phase noise effects: (i) high $\Lambda$ sample resonators together with (ii) low $Q$-value provides a concentration sensitivity equivalent to high $Q$-value resonators. The high-efficiency parameter $\Lambda$ allows the absorption signal of a sample to saturate at low microwave powers, and the low $Q$-value minimizes the demodulation of phase noise by the sample resonator. These sample resonator advances would be adequate if not for the leakage that bypasses the resonator reflection path. Therefore, effort must be made to (iii) decrease this leakage by replacing the circulator with a high-directivity directional coupler and (iv) moving this coupler adjacent to the sample resonator to reduce the difference between the signal and leakage paths. Finally, it is imperative to maintain (v) balanced arms within the bridge to minimize delay-line demodulation of phase noise. We project that these strategies will advance direct detection dispersion mode EPR.

Acknowledgements This work was inspired by the intense effort of Harold M. Swartz to develop portable and robust EPR technology for clinical and preclinical applications.

Funding The research reported in this publication was supported by funding in part by the National Institute of Biomedical Imaging and Bioengineering of the National Institutes of Health under Award No. $\mathrm{P} 41 \mathrm{~EB} 001980$. The content is solely the responsibility of the authors and does not necessarily represent the official views of the National Institutes of Health.

Availability of Data and Material All data is available on request.

\section{Declarations}

Conflict of Interest The authors declare that they have no competing interests.

Open Access This article is licensed under a Creative Commons Attribution 4.0 International License, which permits use, sharing, adaptation, distribution and reproduction in any medium or format, as long as you give appropriate credit to the original author(s) and the source, provide a link to the Creative Commons licence, and indicate if changes were made. The images or other third party material in this article are included in the article's Creative Commons licence, unless indicated otherwise in a credit line to the material. If material is not included in the article's Creative Commons licence and your intended use is not permitted by statutory regulation or exceeds the permitted use, you will need to obtain permission directly from the copyright holder. To view a copy of this licence, visit http://creativecommons.org/licen ses/by/4.0/. 


\section{References}

1. A.M. Portis, Phys. Rev. 91, 1071 (1953). https://doi.org/10.1103/PhysRev.91.1071

2. G. Feher, Phys. Rev. 114, 1219 (1959). https://doi.org/10.1103/PhysRev.114.1219

3. G. Feher, R.A. Isaacson, C.P. Scholes, R. Nagel, Ann. N. Y. Acad. Sci. 222, 86 (1973). https://doi. org/10.1111/j.1749-6632.1973.tb15254.x

4. G. Feher, A.J. Hoff, R.A. Isaacson, L.C. Ackerson, Ann. N. Y. Acad. Sci. 244, 239 (1975). https:// doi.org/10.1111/j.1749-6632.1975.tb41534.x

5. J.S. Hyde, L. Dalton, Chem. Phys. Lett. 16, 568 (1972). https://doi.org/10.1016/0009-2614(72) 80426-3

6. J.S. Hyde, D.D. Thomas, Ann. N. Y. Acad. Sci. 222, 680 (1973). https://doi.org/10.1111/j.17496632.1973.tb15295.x

7. J.S. Hyde, D.D. Thomas, Annu. Rev. Phys. Chem. 31, 293 (1980). https://doi.org/10.1146/annurev. pc.31.100180.001453

8. I. Salikhov, T. Walczak, P. Lesniewski, N. Khan, A. Iwasaki, R. Comi, J. Buckey, H.M. Swartz, Magn. Reson. Med. 54, 1317 (2005). https://doi.org/10.1002/mrm.20689

9. B.B. Williams, R. Dong, A.B. Flood, O. Grinberg, M. Kmiec, P.N. Lesniewski, T.P. Matthews, R.J. Nicolalde, T. Raynolds, I.K. Salikhov, H.M. Swartz, Radiat. Meas. 46, 772 (2011). https://doi.org/ 10.1016/j.radmeas.2011.03.009

10. K.J. Liu, P. Gast, M. Moussavi, S.W. Norby, N. Vahidi, T. Walczak, M. Wu, H.M. Swartz, PNAS 90, 5438 (1993). https://doi.org/10.1073/pnas.90.12.5438

11. C.P. Poole, Electron Spin Resonance: A Comprehensive Treatise on Experimental Techniques, 2nd edn. (Dover Publications, 1996), pp. 250

12. J. Hadbavny, Microw. RF. 24, 91 (1985)

13. E.T. Jaynes, Theory of Microwave Coupling Systems (Naval Research Lab Washington DC Combined Research Group, 1945).

14. T. Moreno, Microwave Transmission Design Data (McGraw-Hill Book Company, 1948).

15. J.R. Ashley, C.B. Searles, F.M. Palka, IEEE Trans. Microw. Theory. 16, 753 (1968). https://doi.org/ 10.1109/tmtt.1968.1126782

16. J.G. Ondria, IEEE Trans. Microw. Theory. 16, 767 (1968). https://doi.org/10.1109/tmtt.1968.11267 84

17. W.P. Robins, Institution of Electrical Engineers, Phase Noise in Signal Sources: Theory and Applications (Peter Peregrinus Ltd, 1982), pp. 53

18. J.W. Sidabras, R.R. Mett, W. Froncisz, T.G. Camenisch, J.R. Anderson, J.S. Hyde, Rev. Sci. Instrum. 78, 034701 (2007). https://doi.org/10.1063/1.2709746

19. J.S. Hyde, W. Froncisz, Advanced EPR, applications, in Biology and biochemistry. ed. by A.J. Hoff (Elsevier, Amsterdam, 1989), pp. 277-306

20. A.I. Tsapin, J.S. Hyde, W. Froncisz, J. Magn. Res. 100, 484 (1992). https://doi.org/10.1016/00222364(92)90055-c

21. G.A. Rinard, R.W. Quine, G.R. Eaton, J. Magn. Res. 144, 85 (2000). https://doi.org/10.1006/jmre. 2000.2014

22. G.A. Rinard, R.W. Quine, J.R. Biller, G.R. Eaton, Concepts Magn. Reson. Part B Magn. Reson. Eng. 37B, 86 (2010). https://doi.org/10.1002/cmr.b.20161

23. G.A. Rinard, R.W. Quine, J. McPeak, L. Buchanan, S.S. Eaton, G.R. Eaton, Appl. Magn. Reson. 48, 1219 (2017). https://doi.org/10.1007/s00723-017-0945-2

24. G.A. Rinard, R.W. Quine, B.T. Ghim, S.S. Eaton, G.R. Eaton, J. Magn. Res. 122, 58 (1996). https:// doi.org/10.1006/jmra.1996.0174

25. J.S. Hyde, W. Froncisz, A. Kusumi, Rev. Sci. Instrum. 53, 1934 (1982). https://doi.org/10.1063/1. 1136918

26. W. Froncisz, T. Oles, J.S. Hyde, Rev. Sci. Instrum. 57, 1095 (1986). https://doi.org/10.1063/1.11386 63

27. J.S. Hyde, M.E. Newton, R.A. Strangeway, T.G. Camenisch, W. Froncisz, Rev. Sci. Instrum. 62, 2969 (1991). https://doi.org/10.1063/1.1142191

28. R. Strangeway, T. Ishii, J. Hyde, Microw. J. 31, 107 (1988)

29. B.F. van der Heyden, IEEE Trans. Microw Theory Tech. 25, 318 (1977). https://doi.org/10.1109/ tmtt.1977.1129094 
30. J.S. Hyde, W. Froncisz, J.W. Sidabras, T.G. Camenisch, J.R. Anderson, R.A. Strangeway, J. Magn. Res. 185, 259 (2007). https://doi.org/10.1016/j.jmr.2007.01.002

31. R.A. Strangeway, J.S. Hyde, T.G. Camenisch, J.W. Sidabras, R.R. Mett, J.R. Anderson, J.J. Ratke, W.K. Subczynski, Cell Biochem. Biophys. 75, 259 (2017). https://doi.org/10.1007/ s12013-017-0804-7

32. J.S. Hyde, R.A. Strangeway, T.G. Camenisch, J.J. Ratke, W. Froncisz, J. Magn. Res. 205, 93 (2010). https://doi.org/10.1016/j.jmr.2010.04.005

33. A.W. Kittell, T.G. Camenisch, J.J. Ratke, J.W. Sidabras, J.S. Hyde, J Magn Reson. 211, 228 (2011). https://doi.org/10.1016/j.jmr.2011.06.004

34. R. Ahmad, S. Som, E. Kesselring, P. Kuppusamy, J.L. Zweier, L.C. Potter, J. Magn. Res. 207, 322 (2010). https://doi.org/10.1016/j.jmr.2010.09.016

35. J. Kozioł, P. Rajda, R. Rumian, T. Oleś, P. Budzioch, R.J. Gurbiel, W. Froncisz, J. Magn. Res. 322, 106870 (2021). https://doi.org/10.1016/j.jmr.2020.106870

36. M. Tseitlin, S.S. Eaton, G.R. Eaton, J. Magn. Res. 209, 277 (2011). https://doi.org/10.1016/j.jmr. 2011.01 .027

Publisher's Note Springer Nature remains neutral with regard to jurisdictional claims in published maps and institutional affiliations. 